\title{
SUGESTÃO DE ALTERNATIVAS DE MATERIAIS DIDÁTICOS PARA O ENSINO DE MICROALGAS
}

\author{
Henrique Varela Adamy ${ }^{1}$, Ana Paula Vestena $\mathrm{Cassol}^{2}$, Camila Elicker ${ }^{3}$, Jéssica Meneghetti ${ }^{4}$, Juliana Ferreira da \\ Silva $^{5}$, Maria Angélica Oliveira ${ }^{6}$
}

\begin{abstract}
${ }^{1}$ Universidade Federal de Santa Maria, Avenida Roraima, 1000 - Camobi, CEP 97105-900, Santa Maria, RS. E-mail: hyck16@gmail.com
${ }^{2}$ Instituto Federal Farroupilha, Rodovia RS-377, s/n - Passo Novo, CEP 97555-000, Alegrete, RS. E-mail: anapvcassol@ gmail.com

${ }^{3}$ Universidade Federal de Santa Maria, Avenida Roraima, 1000 - Camobi, CEP 97105-900, Santa Maria, RS. E-mail:camilaelicker@ hotmail.com ${ }^{4}$ Universidade Federal de Santa Maria, Avenida Roraima, 1000 - Camobi, CEP 97105-900, Santa Maria, RS. E-mail: jessimeneghetti@hotmail.com

${ }^{5}$ Universidade Federal do Pampa, Av. Antonio Trilha, 1847 - São Clemente, CEP 97300-000, , São Gabriel, RS. E-mail: jusferre@ yahoo.com.br

${ }^{6}$ Universidade Federal de Santa Maria, Avenida Roraima, 1000 - Camobi, CEP 97105-900, Santa Maria, RS. E-mail: maria@ufsm.br
\end{abstract}

Resumo - No presente estudo foram confeccionados três materiais didáticos relacionados às microalgas para serem apresentados em turmas de Ensino Fundamental e Médio: jogo da memória de microalgas, moldes tridimensionais e também painéis demonstrando o habitat das mesmas. Para confecção do jogo de memória utilizando cartas de papel cartão e impressão colorida para melhor visualização; para produção dos moldes didáticos utilizando biscuit. Por fim, o painel formado por uma placa de metal e sobre ele colocados os modelos didáticos tridimensionais com imãs colados do lado oposto da estrutura mais marcante da alga representada. $O$ objetivo da confecção desses materiais é facilitar o entendimento de conceitos relacionados às microalgas e de promover uma atenção especial a esse grupo de organismos. Portanto, a utilização de materiais didáticos é de suma importância, sendo uma alternativa útil para familiarizar o aluno com o assunto tratado e também para tornar o estudo de conceitos abstratos algo mais efetivo e atraente.

Palavras-chave: Microalgas. Ensino. Materiais didáticos.

Abstract - The present study made three teaching materials related to microalgae to be presented in elementary school and high school classes: memory game of microalgaes, three-dimensional molds and also panels showing the habitat thereof. For tailoring memory game cards were used paper card and color printing for better viewing; production of didactic molds using biscuit. Finally, a panel formed by a metal plate and on it the three-dimensional didactic models bonded magnets were placed on the opposite side of the most striking structure represented seaweed. The purpose of making these materials is facilitate the understanding of concepts related to microalgae and to promote special attention to this group of organisms. The use of teaching materials is of paramount importance, being a useful alternative to familiarize the student with the subject matter and also to make the study of abstract concepts something more effective and attractive.
Keywords: Microalgae. Education. Teaching Materials.

Recebido em: 09 de dezembro de 2015 Aprovado em: 31 de dezembro de 2015

\section{INTRODUÇÃO}

As microalgas são organismos microscópicos unicelulares predominantemente aquáticos, sendo parte de um grupo bastante diverso de organismos. Podem formar colônias e apresentar pouca ou nenhuma diferenciação celular. Elas produzem mais oxigênio do que todas as plantas do planeta juntas e são responsáveis por cerca de $60 \%$ da produção primária da Terra (CHISTI, 2004).

Segundo Andrade e Costa (2008) e Cristi (2007), microalgas são micro-organismos fotossintéticos que agrupam água, dióxido de carbono e a luz solar para produzir várias formas de energia que serão utilizadas para produção de biomassa, como proteínas, lipídeos, polissacarídeos e hidrocarbonetos. Toda essa biomassa pode ser usada na produção de biocombustíveis e suplementos alimentares e na captura de $\mathrm{CO}_{2}$ da atmosfera.

De acordo com Vidotti e Rollemberg (2004), as microalgas são responsáveis pela estabilidade dos ecossistemas naturais. Microalgas planctônicas, além de serem responsáveis pela produção primária do ambiente aquático, também atuam como indicadores da qualidade ambiental.

As microalgas são identificadas pela sua pigmentação, ciclo de vida e estrutura celular. As diatomáceas (Bacillariophyceae), cianobactérias (Cianophyta), Euglenas (Euglenophyta), algas-verdes (Chlorophyta) e dinoflagelados (Dinophyceae) são os principais grupos de microalgas de águas continentais abordados por Bicudo e Menezes (2006). As diatomáceas são o grupo que domina o fitoplâncton oceânico, mas pode ser encontrada também em água dulcícola. As algas verdes são encontradas tanto nos oceanos quanto na água doce. As algas azuis são encontradas em diversos ambientes, e desempenham papel fundamental na atmosfera através da fixação de oxigênio. As euglenas, que existem tanto em águas marinhas, doces e até no solo, são flageladas 
unicelulares. Os dinoflagelados representam um dos mais importantes grupos do fitoplâncton marinho, conhecidas também pelas marés vermelhas causadas por esse grupo.

Devido à dificuldade de se ensinar certos conceitos da Biologia, especialmente em relação às microalgas, jogos e modelos didáticos podem ser uma alternativa viável para aprimorar o processo de ensino e aprendizagem, sendo vantajoso devido ao baixo custo para produção e pelo fato de serem adaptados pelos próprios alunos, visto que são jogos manuais. Todos esses aspectos proporcionam uma maior assimilação do conteúdo por parte dos estudantes (COELHO et al, 2010). Embora existam ferramentas de ensino inovadoras como informática, multimídia, internet, os professores ainda sentem dificuldade em sala de aula devido à desmotivação dos alunos em relação à aprendizagem. Fialho (2008) destaca que os jogos são instrumentos motivadores e de grande potencial de sociabilidade e interação no que se refere ao processo de ensino e aprendizagem.

Uma crítica recorrente se deve ao fato de os docentes geralmente basearem- se em um ensino teórico e relacionado ao livro didático, sendo os conteúdos estudados muitas vezes dissociados da vida prática do estudante. As modalidades de ensino tradicionais baseadas em aulas expositivas ainda prevalecem, de forma que novidades tecnológicas e novas ferramentas de estudo ainda são pouco desenvolvidas nas escolas (SILVA; VALLIM, 2015).

Em um estudo feito por Silva e Giordani (2009) pode-se observar que a maioria dos docentes acredita que os materiais didáticos são de suma importância, uma vez que contribuem para a aquisição de novos conhecimentos. Os educadores acreditam que a teoria é muito importante para os alunos, mas junto a ela devese dispor de instrumentos reais e concretos para que o aprendizado do aluno seja significativo. Assim, é necessário adotar instrumentos pedagógicos, tais como jogos didáticos, para tornar o estudo de conceitos abstratos mais efetivos e atraentes. Os jogos, por desenvolverem o aspecto lúdico e a dinamização, podem colaborar para que o aluno aprenda melhor os conceitos estudados (PAVAN, 1998).

\section{OBJETIVO}

O presente trabalho teve como objetivo apresentar sugestões de materiais didáticos para auxiliar no ensino de microalgas para escolas de Ensino Fundamental e Médio, para promover integração entre alunos e professores, levando a uma aprendizagem mais significativa.

\section{MATERIAIS E MÉTODOS}

Três materiais de uso didático de fácil obtenção e produção com preço acessível são sugeridos neste trabalho. Um jogo de cartas do tipo jogo da memória com fotos de diferentes gêneros dos principais grupos de microalgas, modelos tridimensionais dispostos em um painel de metal representando três habitas típicos de microalgas.

\subsection{Jogo da memória das microalgas}

Para o jogo da memória das microalgas, foram confeccionadas cartas em papel cartão, mais resistente e com impressão colorida. Cada carta contém um gênero de alga estampado e identificado. O jogo apresenta 18 pares de cartas (Figura 1), e pode ser jogado por até quatro jogadores.

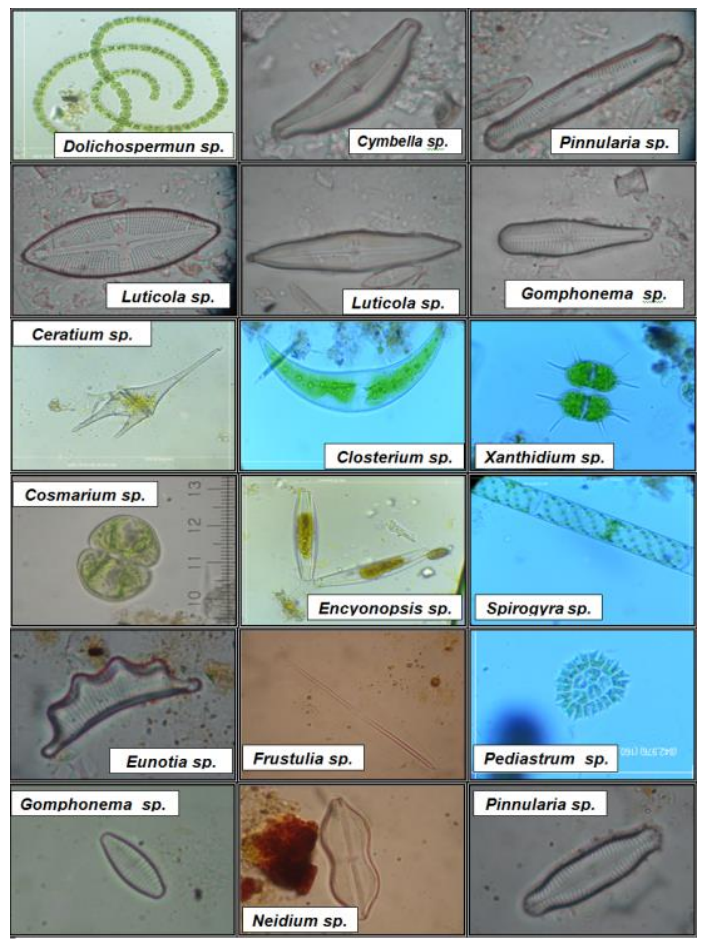

Figura 1 - Cartas do jogo da memória.

O jogo funciona de maneira simples, o professor deve dividir os alunos em grupos de quatro, cada grupo com um baralho de 36 cartas. Em cada grupo as cartas serão embaralhadas e postas com a foto das microalgas voltada para baixo, cada participante tem que virar duas cartas, quando as cartas formarem um par, ele faz um ponto e recolhe as cartas e tem direito de tentar mais uma vez, quando as cartas não formarem um par o participante perde a vez. O jogo termina quando todas as cartas forem coletadas, o participante com mais cartas acumuladas, ganha o jogo.

\subsection{Modelos didáticos}

Os modelos tridimensionais foram feitos em biscuit, utilizando fotos e desenhos de microalgas que são informações para a confecção dos modelos que devem ter representados as principais estruturas da microalga.

\subsection{Painel dos habitats das microalgas}


O painel foi formado por uma placa de metal e os modelos didáticos tridimensionais com imãs colados na parte oposta da estrutura mais marcante da alga representada (Figura 2 e 3), para serem colocados no painel.

\section{RESULTADOS E DISCUSSÃO \\ 4.1 Jogo da memória das microalgas}

Os jogos de memória além de promoverem uma integração entre o grupo participante pode ser utilizado como uma ferramenta de ensino e assimilação dos conteúdos. Este jogo permite um contato com diversidade de espécies de microalgas encontradas em águas continentais, proporcionando muitas vezes um primeiro contato com esses micro-organismos, pois muitos não sabem nem mesmo da existência delas.

\subsection{Modelos didáticos}

$\mathrm{Na}$ aplicação em uma aula dos modelos didáticos, alunos e professores se reuniriam para confeccionar os modelos de microalgas, dos principais grupos de microalgas e destacando as estruturas mais marcantes de cada grupo, baseando-se em imagens e descrições. Além da integração, possibilita uma maior visualização de estruturas características de cada grupo/espécie microalgal.

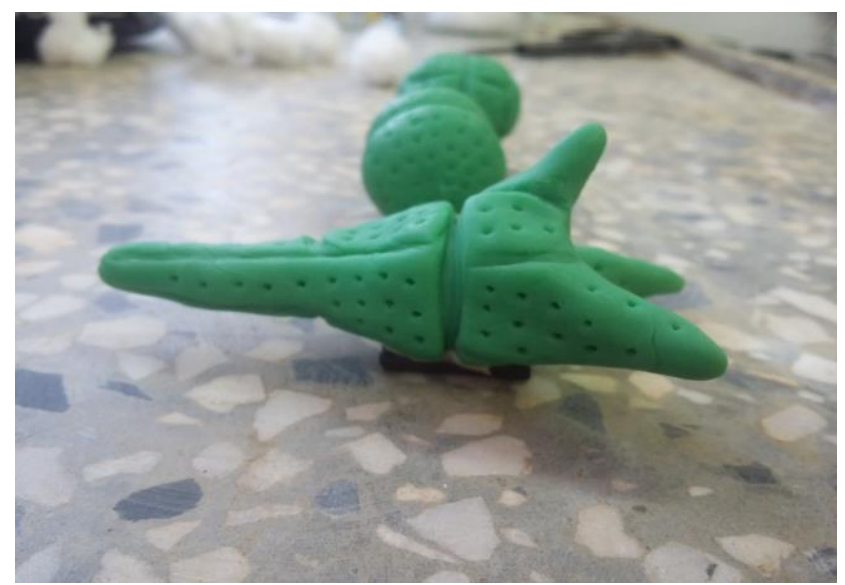

Figura 2 - Modelo didático representando um dinoflagelado, com imã na parte oposta às características que foram destacadas.

\subsection{Painel dos habitats das microalgas}

$\mathrm{Na}$ parte superior do painel representa uma macrófita aquática, feita de no acetato de vinila colorido, conhecido como popularmente como espuma vinílica acetinada (EVA). A coluna d'água ilustrada em azul claro, e o leito do corpo hídrico também foi feito de EVA, imitando rochas. Os modelos didáticos colocados juntos à macrófita e ao leito do corpo hídrico representam o perifíton, que é a comunidade de microrganismos que vive aderida a substratos inorgânicos ou orgânicos, vivos ou mortos (WETZEL, 1983).
Os modelos dispostos longe da macrófita e do leito representam o fitoplâncton, os organismos fotossintetizantes que são adaptados a viver livres em suspensão em águas abertas, toda a vida ou parte dela (REYNOLDS, 2006). A facilidade de transmissão de conteúdo, tais como este, proposto através de jogos favorece a aprendizagem de conceitos abstratos e complexos, além de proporcionar motivação interna, o raciocínio, a argumentação, a interação entre alunos e professores (CAMPOS, 2003).

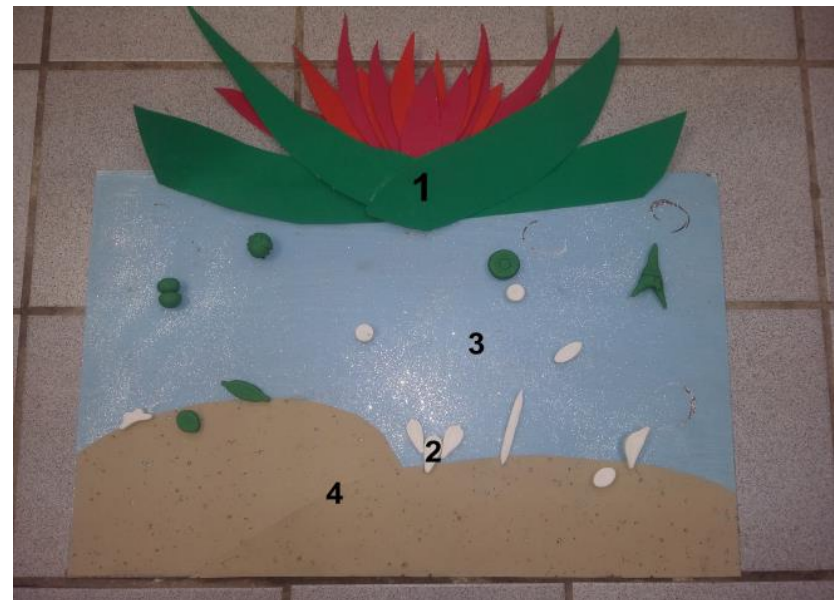

Figura 3 - Imagem do Painel dos habitats das microalgas. (1) Macrófita Aquática (Epifíton), (2) Organismos do perifíton, (3) Coluna d'agua, (4) Sedimentos do fundo do corpo hídrico (Epiliton).

Brotto (2002) relata que em função dessas possibilidades que os jogos proporcionam, dando destaque para os jogos cooperativos, nos quais os objetivos são comuns, as ações são compartilhadas e os benefícios são distribuídos para todos . Adicionalmente, é importante aproveitá-los como uma alternativa para a diminuição da violência, falta de disciplina e evasão escolar.

Os jogos didáticos são uma atividade física e mental que desenvolvem diferentes capacidades. Por isso é uma ótima ferramenta no processo educativo. Melo (2008) destaca que jogos em sala de aula, o aluno aprende inconscientemente, pois a diversão alivia a pressão escolar e a necessidade constante de dominar o conteúdo. Esta pressão escolar passa a ser substituída pela descontração e os resultados são mais expressivos. Segundo Soares (2008), há muitos trabalhos relacionados a esse instrumento pedagógico, principalmente, nas áreas de Matemática, Física e Biologia/Ciências. Talvez, no caso das Ciências e a Biologia, isso se deva por serem áreas do conhecimento que tratam de conteúdos abstratos, de difícil compreensão muitas vezes, e que ainda hoje sofrem com abordagens tradicionais no ensino, isso quando são abordadas. Ainda prevalecem a transmissão-recepção de informações (CAMPOS et al, 2003). Assim o uso de jogos seria uma tentativa de melhorar a construção desses conhecimentos. 


\section{CONCLUSÃO}

Esse tipo de material didático é algo que promove uma interação entre aluno-aluno e aluno-professor. Auxilia na aprendizagem de um assunto muitas vezes de difícil compreensão como os relacionados às microalgas. Adicionalmente, espera-se que esta iniciativa seja exemplo para demais temas, tornando o estudo de Ciências e/ou Biologia mais interessante e produtivo.

\section{REFERÊNCIAS}

ANDRADE, M. R.; COSTA, J. A. V. Cultivo da microalga Spirulina platensisem fontes alternativas de nutrientes. Ciência e Agrotecnologia, Lavras, v. 32, n. 5, p. 1551-1556, 2008.

BICUDO, C. E. M.; MENEZES, M. Gêneros de algas de águas continentais do Brasil. 2.ed. São Carlos. RiMa. 2006.

BROTTO, F.O. Jogos Cooperativos: o jogo e o esporte como um exercício de convivência. Santos: Projeto Cooperação, 2002.

CAMPOS, L. M. L.; FELICIO, A. K. C.; BORTOLOTTO, T. M. A produção de jogos didáticos para o ensino de Ciências e Biologia: uma proposta para favorecer a aprendizagem. Caderno dos Núcleos de Ensino, p. 35-48, 2003.

CHISTI, Y. Microalgae: our marine forests. In: RICHMOND, A. (Ed). Handbook of microalgal culture: biotechnology and applied phycology. Oxford: Blackwell Science, 2004. 566 p.

COELHO, F. S. et al. Jogos e modelos didáticos como instrumentos facilitadores para o ensino de biologia. In: SEMINÁRIO DE EXTENSÃO DA PUC MINAS, 5. 2010, [S.1.], [Anais...], 2010.
FIALHO, N. N. Os jogos pedagógicos como ferramentas de ensino. In: CONGRESSO NACIONAL DE EDUCAÇÃO, 8., 2008, Curitiba. [Anais...] Curitiba: PUCPR, 2008

MELO, A.V.F. Jogo pedagógico, Brasil e sua

dinâmica territorial: educação lúdica em geografia. Universidade Cruzeiro do Sul: Pelotas, 2008.

PAVAN, O. H. O. et al. Evoluindo genética: um jogo educativo. Campinas: Unicamp, 1998.

REYNOLDS, C. S. The ecology of phytoplankton. Cambridge: University Press., 2006.

SILVA, E.L; GIORDANI, E.M. Aprendizagens de professores e alunos com materiais didáticos nos anos iniciais do ensino fundamental. In: CONGRESSO

NACIONAL DE EDUCAÇÃO, 9., Curitiba, 2009. [Anais...], Curitiba, 2009.

SILVA, J. B.; VALLIM M. A. Estudo, desenvolvimento e produção de materiais didáticos para o ensino de biologia. Revista Aproximando, v. 1, n.1, 2015.

SOARES, M. H. F. B. Jogos e Atividades Lúdicas no Ensino de Química: Teoria, Métodos e Aplicações. In: ENCONTRO NACIONAL DE ENSINO DE QUÍMICA, 14., 2008, Curitiba. [Anais...], Curitiba: UFPR, 2008.

VIDOTTI, E. C.; ROLLEMBERG, M.C.E. Algas: da economia nos ambientes aquáticos à bioremediação e a química analítica. Química Nova, v. 27, n.1, p. 139 145, 2004.

WETZEL, R. G. Lymnology. 2nd. Ed. New York: Saunders College Publishing. 1983. 